\title{
Focal seizures with impaired awareness as long-term neurological complication of COVID-19: a case report
}

\author{
Marco Bozzali ${ }^{1,2}$ (1) - Alberto Grassini ${ }^{1}$ - Giovanni Morana ${ }^{1} \cdot$ Michela Zotta $^{3} \cdot$ Sara Cabras $^{1} \cdot$ Alberto Romagnolo $^{1}$. \\ Carlo Alberto Artusi ${ }^{1}$ - Elisa Montalenti ${ }^{4}$. Mario Giorgio Rizzone ${ }^{1} \cdot$ Diego Garbossa ${ }^{1} \cdot$ Elisa Montanaro ${ }^{4}$. \\ Mara Cercignani $^{2} \cdot$ Leonardo Lopiano $^{1,4}$
}

Received: 17 January 2021 / Accepted: 6 April 2021 / Published online: 16 April 2021

(C) Fondazione Società Italiana di Neurologia 2021

\begin{abstract}
We report here the first case of a young individual otherwise healthy, who presented with frequent focal seizures with impaired awareness as a possible long-term complication of severe acute respiratory syndrome coronavirus-2 infection. Seizures were documented by electroencephalography and responded clinically and neuro-physiologically to antiseizure therapy. The patient underwent an extensive investigation including cerebrospinal fluid examination, conventional and quantitative brain magnetic resonance imaging, and 18-FDG positron emission tomography. Beyond the clinical interest, this case contributes to clarify the possible pathways by which SARS-CoV-2 may enter the central nervous system and cause long-term neurological complications.
\end{abstract}

Keywords COVID-19 $\cdot$ Epilepsy $\cdot$ Focal seizures with impaired awareness $\cdot$ Encephalitis

\section{Introduction}

First-ever occurrence of seizures in non-epileptic individuals have been described in the acute phase of severe acute respiratory syndrome coronavirus-2 (SARS-CoV-2) infection [1]. In these cases, possible underlying mechanisms include metabolic or fever induced brain dysfunction, cytokine-storm damage, brain vessel endothelial infarction, autoimmunity, or central nervous system (CNS) viral invasion [2]. We report here the case of a young individual with no remarkable medical history who presented with frequent focal seizures with impaired awareness newly arisen 2 months after resolution of their coronavirus disease 2019 (COVID-19) acute phase.

Marco Bozzali

marco.bozzali@unito.it

1 Department of Neuroscience "Rita Levi Montalcini", University of Torino, Via Cherasco 15, 10126 Turin, Italy

2 Department of Neuroscience, Brighton \& Sussex Medical School, University of Sussex, Brighton, East Sussex, UK

3 Department of Diagnostic Imaging, Nuclear Medicine Unit, A.O.U. Città della Salute e della Scienza di Torino, Turin, Italy

4 Neurology 2 Unit, A.O.U. Città della Salute e della Scienza di Torino, 10124 Turin, Italy

\section{Case report}

A 54-year-old lady working as a nurse in a COVID-19 ward received, at the end of March 2020, her first-ever SARS-CoV-2 nasopharyngeal swab after reporting a high-risk contact with a colleague suffering from COVID-19. Despite a negative outcome, 5 days later, she started complaining of fever, nasal congestion, throat pain, fatigue, shortness of breath, muscle and joint pain, diarrhoea, anosmia, and ageusia. These symptoms persisted for 4 weeks while she remained at home in precautionary isolation. At the end of April 2020, she recovered from all symptoms with the exception of fatigue, muscle and joint pain, anosmia, and ageusia. Concomitantly, she started complaining of continuous headaches and feeling of gait unbalance. She was admitted to the emergency ward of the University Hospital of Turin and received full blood and arterial blood oxygenation tests (all parameters in normal range), chest X-rays (normal), and real-time polymerase chain reaction (PCR) on nasopharyngeal swab (positive for SARS-CoV-2). Due to her clinical stability, she was discharged and remained at home in quarantine until she resulted negative on two consecutive SARS-CoV-2 swab tests. She was serologically tested for antibodies antiSARS-CoV-2, resulting IgG positive and IgM negative. Clinically, she kept complaining of fatigue, gait unbalance, and headaches. In June 2020, she underwent a brain MRI scan, 


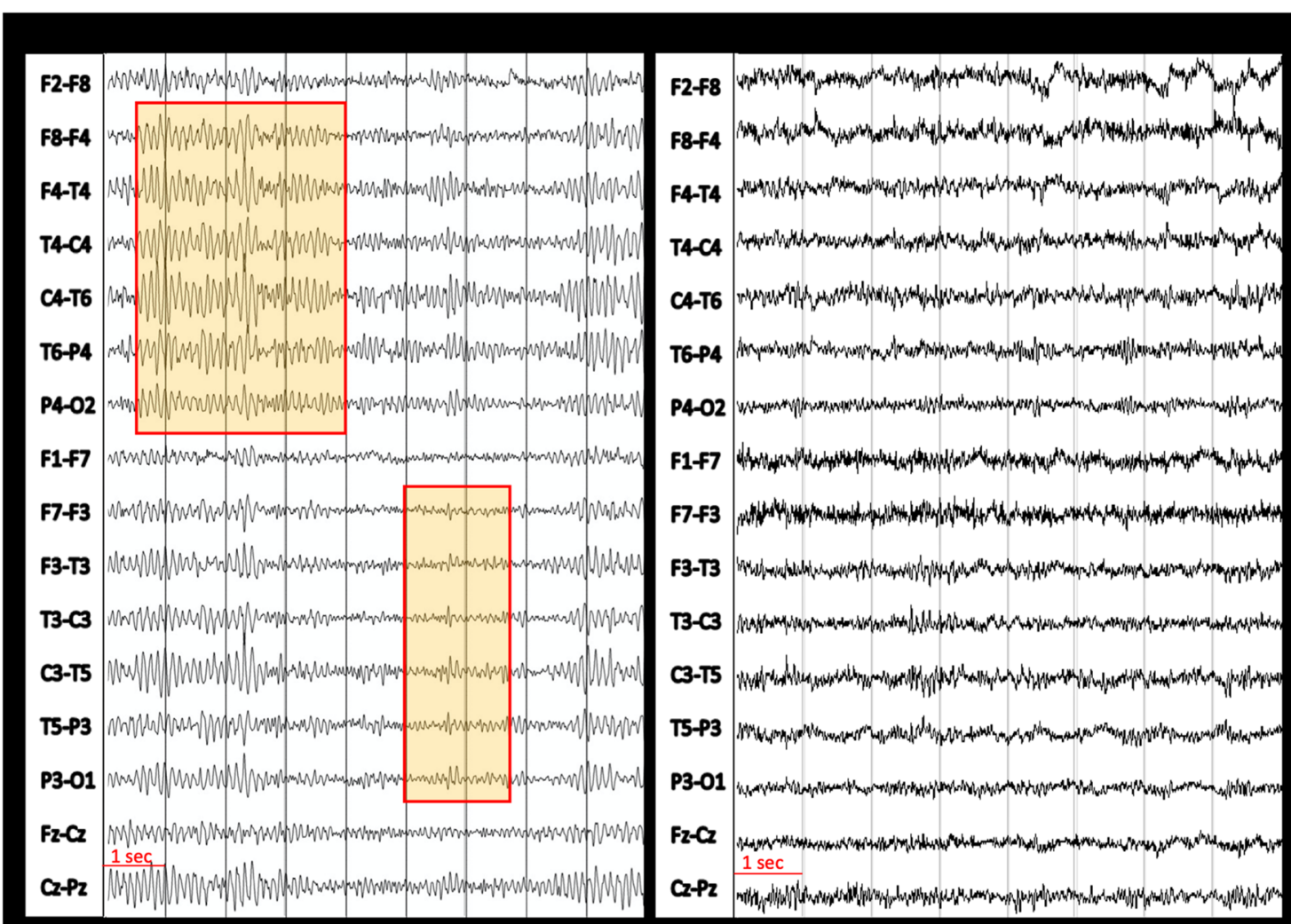

a

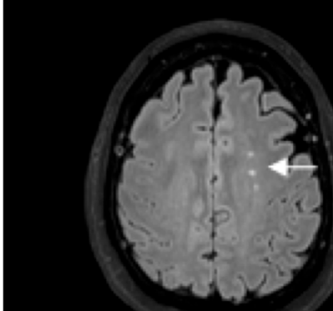

C

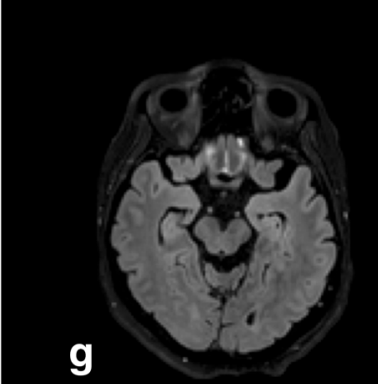

d

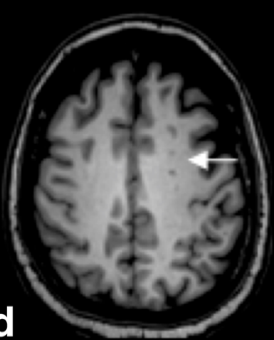

b

e
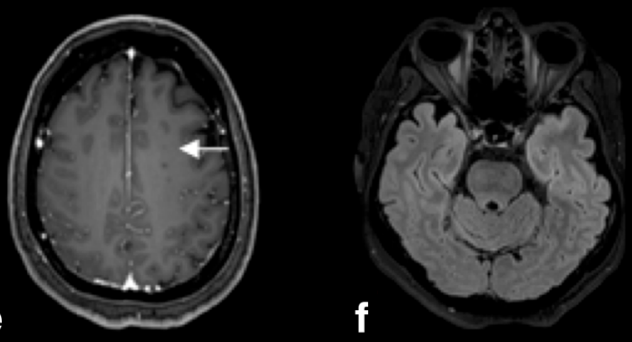

$\mathbf{h}$

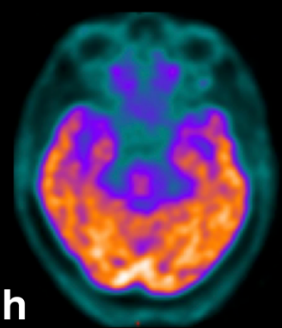

i

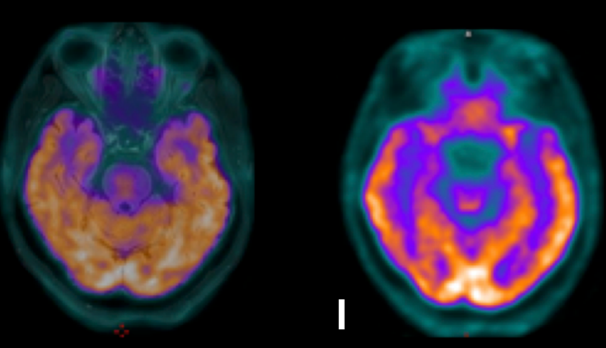

Fig. 1 The first EEG, recorded before therapy initiation, showed a pattern of focal slow activity and spikes in the fronto-temporal area bilaterally (abnormalities magnified in red squares) (a). The second EEG, performed 4 weeks after initiation of antiseizure medication, did no longer reveal any pathological alteration (b). EEG recordings were performed with scalp electrodes placed according to the international 10-20 system with bipolar montage; brain MRI at the level of the centrum semiovale

demonstrated only few hyperintense foci on FLAIR (c), mildly hypointense on T1-weighted images (d) with no contrast enhancement (CE) on corresponding CE T1-weighted images (e). These abnormalities keep with minimal non-specific changes (arrow, c-e). Co-registered FLAIR (f, g), 18F-FDG PET (h, l), and fused PET/MRI FLAIR images (i) did not show any temporal lobe abnormalities, with physiological tracer uptake according to patient's age

showing minimal non-specific white matter hyperintensities on T2-weighted images. In July 2020, she started complaining of frequent (6-8 times a day) short episodes (about 1-min duration each) of olfactory hallucinations (described as "burning rubber 


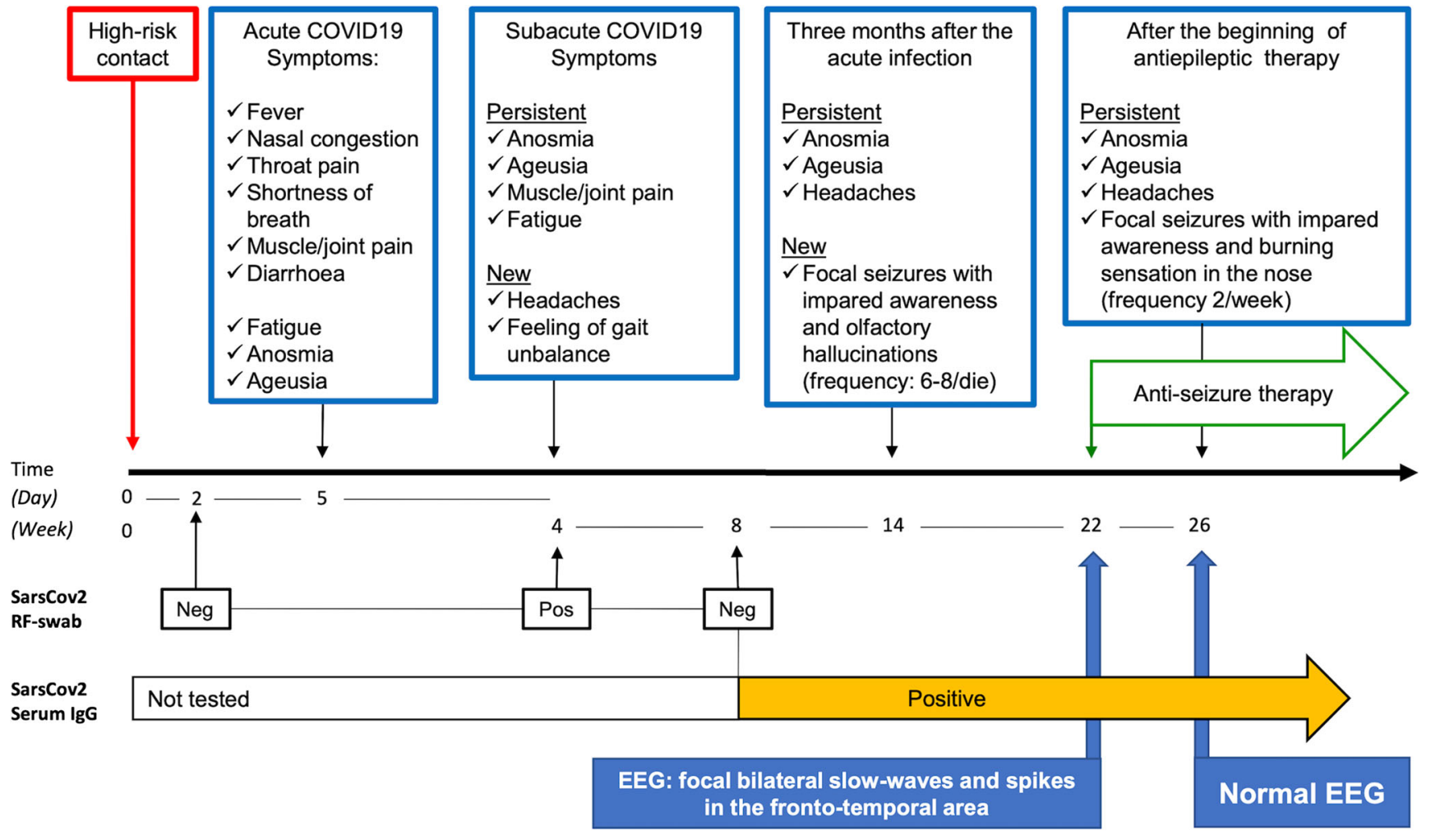

Fig. 2 The picture summarizes the clinical evolution of SARS-CoV-2 infection in the presented case alongside findings from the most relevant laboratory and instrumental examinations. See text for further details

smell") followed by 10-15-min intervals of detachment from reality (described as "mental confusion"). In mid-September 2020, she was admitted to our Neurology Unit (Department of Neuroscience, University of Turin, Italy) for investigation. On examination, there were no obvious impairments in her higherlevel functions; cranial nerves were intact; there was no weakness at any limb; muscular tone was normal; reflexes were symmetrical and plantars downgoing bilaterally; coordination tests were well performed; there were no sensory deficits; her gait was normal. A mild right deviation was observed on the Unterberger test. She underwent an electroencephalogram (EEG) demonstrating the presence of focal slow waves alongside sharp spikes in the fronto-temporal areas bilaterally (Fig. 1a). Repetition of SARS-CoV-2 nasopharyngeal swab was negative, while serum-specific SARS-CoV-2 IgG remained highly positive $(140 \mathrm{U} / \mathrm{ml})$. Blood tests, including vitamin B12, folates, and thyroid function tests, were normal. Serological analysis to exclude autoimmune diseases and systemic infection resulted negative. A new brain MRI scan at 3T, including highresolution conventional acquisitions (FLAIR, T1-, T2-weighted, susceptibility-weighted imaging, and post-contrast T1-weighted images), confirmed prior non-specific white matter changes (no additional abnormalities) (Fig. 1c-g). CSF examination revealed a slight increase of proteins $(55 \mathrm{mg} / \mathrm{dl})$ with normal cell count. CSF PCR for detection of common neurotropic pathogens (i.e. Herpes simplex 1 and 2, Human herpes 6, Enterovirus, human parechovirus, Varicella zoster, Cytomegalovirus,
Streptococcus pneumoniae, Neisseria meningitidis, Haemophilus influenzae, Streptococcus agalactiae, Listeria monocytogenes, Escherichia coli K1, Cryptococcus neoformans) and for SARS-CoV-2 returned negative results. Immunoelectrofocusing did not reveal any oligoclonal bands. No specific antibodies anti-SARS-CoV-2 neither brain autoimmune (anti-NMDA glutamate receptor, AMPA 1 and 2 glutamate receptor, GABA receptor, LG1, CASPR2, DPPX, thyroperoxidase, thyroglobulin, TSH receptor) nor paraneoplastic antibodies (anti-YO, Sox1, Zic4, Titin, Hu, GAD65, CV2, Ri, Ma2, Retin, Amphiphysin, Tr) were identified on the CSF. Considering the feeling of gait unbalance and deviation on the Unterberger test, she underwent also brainstem auditory evoked potentials (BAEP) showing a mild bilateral increase of latencies. An extensive neuropsychological assessment revealed an isolated impairment of frontal-executive functions (frontal assessment battery corrected score $=12.3$ ). For this reason, the patient underwent a brain $\left[{ }^{18} \mathrm{~F}\right] \mathrm{FDG}$ positron emission tomography that resulted normal (Fig. 1h-1). She was started on levetiracetam therapy with only partial benefit and then shifted to carbamazepine, $400 \mathrm{mg}$ twice a day. After being on carbamazepine for 12 weeks, the patient reported clear improvement of symptoms with "burning sensation" [3] in her nose (instead of smell hallucinations) occurring with reduced frequency ( 2 times a week) alongside shorter intervals of detachment from reality. Moreover, EEG repetition did no longer show any spikes and revealed reduction of slow waves (Fig. 1b). 


\section{Discussion}

Since COVID-19 became clinically overt, the patient presented with a clinical progression that fits with a subacute encephalitis (Fig. 2). Despite lack of evidence of SARS-CoV-2 RNAs in the CSF, the clinical evolution indicates a plausible anatomical pathway by which SARS-CoV-2 may have subacutely infected the central nervous system (CNS). Being primarily a respiratory virus, there are at least two possible ways for SARS-CoV-2 to invade the CNS, through an haematogenous or an axonal route [4]. In the latter case, SARS-CoV-2 is supposed to infect the axonal terminations and actively translocate backwards into the CNS. The neuronal fibres that are most likely infected by SARS-CoV-2 are those of the olfactory nerves, as suggested by anosmia as typical symptom of COVID-19 and supported by radiological evidence [5]. Another possible route is trough other cranial nerves with invasion of the brainstem, as partially supported by our clinical (Unterberger test) and neurophysiological (BAEP) observations. Evidence of paracellular transmigration of betacoronaviruses (e.g. SARS-CoV-1) has been provided in animal studies, with viral demonstration within the olfactory bulb, piriform cortex, hippocampus and temporo-mesial cortex, thalamus, and brainstem nuclei $[6,7]$. Importantly, persistence of coronavirus RNAs in the CNS was demonstrated long time after acute encephalitis [8]. In our patient, we were unable to detect viral RNAs in the CSF. This might be due to the long interval elapsed between the acute COVID-19 phase and CSF examination. Consistently, it was previously shown that SARS-CoV-2 RNAs are detectable in the CSF of a small percentage of COVID-19 patients with neurological symptoms [9], often requiring repeated CSF examination [10]. Nonetheless, our patient developed focal seizures with impaired awareness (documented by EEG) responding to antiseizure medication. In contrast to previous reports in acute cases $[10,11]$, we could not demonstrate abnormalities in the temporo-mesial cortex, which — we argue - might no longer be detectable in post-acute COVID-19 stages. On neuropsychological assessment, our patient showed an isolated deficit of executive functions, whose relationship with SARS-CoV-2 infection remains to be clarified despite a suggestive anatomical overlap with fronto-temporal seizures.

In conclusion, we report here a case of fronto-temporal epilepsy, possibly related to SARS-CoV-2 infection. This diagnosis of causality, which remains speculative, is based on exclusion assessments and clinical elements supporting such a hypothesis (clinical evolution, EEG alterations; response to antiseizure medication).

We expect this sort of long-term complications requiring immediate identification and treatment to become increasingly frequent in the future.
Author contribution M.B., A.G., and L.P. planned all clinical and instrumental investigations, interpreted the results, and wrote the first draft of the manuscript. G.M. and M.C. supervised MRI acquisition and contributed to data interpretation. M.Z. supervised PET imaging and contributed to data interpretation. A.R., M.G.R., C.A.A., D.G., and S.C. performed clinical assessments and managed all serological and CSF examinations. E. Montal. contributed to interpretation of neurophysiological data. E. Montan. did neuropsychological testing. All Authors were involved in drafting or revising the manuscript.

Data availability MB and AG have full access to all data presented in the study and take responsibility for their integrity and for the accuracy of data analysis.

\section{Declarations}

Ethical approval All procedures were performed in accordance with the local ethics committee and with the ethical standards laid down in the 1964 Declaration of Helsinki and its later amendments.

Conflict of interest The authors declare no competing interests.

Informed consent The patient provided informed written consent.

\section{References}

1. Mao L, Wang M, Chen S et al (2020) Neurological manifestations of hospitalized patients with COVID-19 in Wuhan, China: a retrospective case series study. JAMA Neurol. https://doi.org/10.1001/ jamaneurol.2020.1127

2. Asadi-Pooya AA (2020) Seizures associated with coronavirus infections. J Seizure. https://doi.org/10.1016/j.seizure.2020.05.005

3. Heo K, Kim KM, Han SM, Cho KH, Chu MK (2020) Nasal pain as an aura: Amygdala origin? Seizure 2020:13-16. https://doi.org/10. 1016/j.seizure.2020.09.028

4. Mishra R, Banerjea A (2020) Neurological damage by coronaviruses. Front Immunol. https://doi.org/10.3389/fimmu. 2020.565521

5. LaurendonT RT, Mugnier J et al (2020) Bilateral transient olfactory bulb edema during COVID-19-related anosmia. Neurology. 95: 224-225. https://doi.org/10.1212/wnl.0000000000009850

6. Pearlman S, Jacobsen G, Afifi A (1989) Spread of a neurotropic murine coronavirus into the CNS via the trigeminal and olfactory nerves. Virology. https://doi.org/10.1016/0042-6822(89)90446-7

7. McCray PB, Pewe L, Wohlford-Lenane C et al (2007) Lethal infection of K18-hACE2 mice infected with severe acute respiratory syndrome coronavirus. J Virol 81:813-821. https://doi.org/10. 1128/jvi.02012-06

8. Jacomy H, Fragoso G, Almazan G, Mushynski WE, Talbot PJ (2006) Human coronavirus OC43 infection induces chronic encephalitis leading to disabilities in BALB/C mice. Virology. 349: 335-346. https://doi.org/10.1016/j.virol.2006.01.049

9. Neumann B, Schmidbauer ML, Dimitriadis K et al (2020) Cerebrospinal fluid findings in COVID-19 patients with neurological symptoms. J Neurol Sci. https://doi.org/10.1016/j.jns.2020. 117090

10. Virhammar J, Kumlien E, Fällmar D, Frithiof R, Jackmann S, Sköld MK, Kadir M, Frick J, Lindeberg J, Olivero-Reinius H, Ryttlefors M, Cunningham JL, Wikström J, Grabowska A, Bondeson K, Bergquist J, Zetterberg H, Rostami E (2020) Acute necrotizing 
encephalopathy with SARS-CoV-2 RNA confirmed in cerebrospinal fluid. Neurology. 95:445-449. https://doi.org/10.1212/WNL. 0000000000010250

11. Moriguchi T, Harii N, Goto J, Harada D, Sugawara H, Takamino J, Ueno M, Sakata H, Kondo K, Myose N, Nakao A, Takeda M, Haro H, Inoue O, Suzuki-Inoue K, Kubokawa K, Ogihara S, Sasaki T, Kinouchi H, Kojin H, Ito M, Onishi H, Shimizu T, Sasaki Y, Enomoto N, Ishihara H, Furuya S, Yamamoto T, Shimada S
(2020) A first case of meningitis/encephalitis associated with SARS-Coronavirus-2. Int J Infect Dis. 94:55-58. https://doi.org/ 10.1016/j.ijid.2020.03.062

Publisher's note Springer Nature remains neutral with regard to jurisdictional claims in published maps and institutional affiliations. 